\title{
Evaluation of the human sperm acrosome reaction using a monoclonal antibody, GB24, and fluorescence-activated cell sorter
}

\author{
P. Fénichel*†, B. L. Hsi*, D. Farahifar*, M. Donzeau $\dagger$, D. Barrier-Delpech $\dagger$ \\ and C. J. G. Yeh* \\ *INSERM U210, Faculté de Médecine, 06034 Nice Cedex, France; and \\ $\uparrow$ Laboratoire de Biologie de la Reproduction, Faculté de Médecine, Nice, France
}

\begin{abstract}
Summary. GB24 is a mouse monoclonal antibody raised against human trophoblast microvilli, which recognizes an antigenic determinant on the acrosomal region of the human sperm head. By indirect immunofluorescence, reactivity of GB24 could not be detected on freshly ejaculated spermatozoa but was strongly positive after sperm permeabilization with acetone. On viable, motile spermatozoa, reactivity appeared after induction of the acrosomal reaction with the calcium ionophore A23187. These results suggest that the antigen recognized by GB24 is present on the inner acrosomal membrane. A quantitative evaluation assay of the acrosome reaction on viable spermatozoa by flow cytometry using GB24 and indirect immunofluorescence is proposed.
\end{abstract}

Keywords: human; acrosome reaction; monoclonal antibody; inner acrosomal membrane; fluorescenceactivated cell sorter

\section{Introduction}

Capacitation and the acrosome reaction are prerequisites for fertilization by human spermatozoa. Several physiological and pathophysiological aspects of these processes are not yet fully understood, one reason being the lack of a rapid and suitable clinical assay. Capacitation occurs in vivo in the female genital tract and can be induced in different culture media used for in-vitro fertilization (Bedford, 1983). This process, which involves modification of plasma membrane components on the sperm head, can be indirectly monitored by the zona-free hamster egg penetration test (Yanagimachi et al., 1976). The acrosome reaction takes place in vivo around the oocytes and can be induced in vitro with the calcium ionophore A23187 (Jamil \& White, 1981). Although the morphological exocytotic events of the acrosome reaction have been well documented by electron microscopy (Barros et al., 1967), it is difficult to observe the human acrosome reaction by optical microscopy. A triple staining technique on fixed spermatozoa has been proposed for this purpose (Talbot \& Chacon, 1981).

It has been suggested that monoclonal antibodies raised against sperm antigens could be used as immunomarkers to follow the biochemical modifications and stages of sperm maturation (Wolf et al., 1985; Kallajoki et al., 1986). Of the several mouse monoclonal antibodies raised against human trophoblast in our laboratory (Hsi \& Yeh, 1986; Hsi et al., 1987), one, GB24, was found to recognize a common antigen on trophoblast and lymphocytes (Hsi et al., 1988). We report here the use of GB24 and flow cytometry to evaluate sperm capacitation and the acrosome reaction. 


\section{Materials and Methods}

Sperm preparation. Sperm samples from proven fertile donors or from husbands participating in an in-vitro fertilization programme were used with the following standards: $>50 \times 10^{6}$ spermatozoa $/ \mathrm{ml} ;>70 \%$ motile spermatozoa; $>70 \%$ normal forms. Viable and motile spermatozoa were collected using a 'swim-up' technique. After 30 min liquefaction at $37^{\circ} \mathrm{C}$, spermatozoa were washed twice in Tyrode's medium (Eurobio, Paris, France), centrifuged at $500 \mathrm{~g}$ for $10 \mathrm{~min}$, then allowed to swim-up for $30 \mathrm{~min}$, in B2 medium (Ménézo, 1976; Api system, Montalieu, France) containing $1 \%$ bovine serum albumin and $1.75 \mathrm{~mm}-\mathrm{CaCl}_{2}$. Viable motile spermatozoa were subsequently collected from the supernatant and the final sperm concentration in B2 medium was adjusted to $1-10 \times 10^{6} / \mathrm{ml}$. Motility and viability were determined by phase-contrast microscopy and Trypan blue dye exclusion. Samples were placed on slides, air-dried and subsequently fixed with acetone for $10 \mathrm{~min}$.

Induction of sperm capacitation and the acrosome reaction. Motile viable spermatozoa were incubated in B2 medium at $37^{\circ} \mathrm{C}$ in $5 \% \mathrm{CO}_{2}, 95 \%$ air for $6 \mathrm{~h}$ to induce capacitation. Then, the acrosome reaction was induced by incubating the spermatozoa with the calcium ionophore A23187 (Sigma, St Louis, MO, USA), at final concentrations of $1-20 \mu \mathrm{M}$, in $\mathrm{B} 2$ medium for 30,60 and $120 \mathrm{~min}$. The calcium ionophore was dissolved in ethanol to a concentration of $2 \mathrm{~mm}$ and stored at $-20^{\circ} \mathrm{C}$.

Antibodies. GB24 was derived from the fusion of mouse myeloma P3-NS1/1-Ag4-1(NS-1) cells (Flow Laboratories, Irvine, UK) and spleen cells of a mouse which was immunized with placental syncytiotrophoblast microvilli prepared by differential ultracentrifugation (Hsi et al., 1988). The isotype of GB24 is IgG1 kappa. For indirect immunofluorescence, supernatants of GB24 were first used for antigen localization. For direct immunofluorescence, the IgG of GB24 was purified by caprilic acid precipitation (Russo et al., 1983) and subsequently conjugated with fluorescein isothiocynate (FITC) according to the method of Johnson \& Holborow (1986) at a final fluorescein:immunoglobulin ratio of 2:1. Two monocional antibodies (IgG1) were used as controls, GB3 (Verrando et al., 1987) for indirect immunofluorescence and FITC-conjugated IOT8 (Immunotech, Marseille, France) for direct immunofluorescence.

Immunofuorescence. For indirect immunofluorescence, pooled and tested supernatants of GB24 were used. Spermatozoa were incubated with GB24 at 1/10 dilution in B2 medium for $30 \mathrm{~min}$, washed in phosphate-buffered saline (PBS, $0.15 \mathrm{M}, \mathrm{pH} 7$ ), then incubated with FITC-conjugated rabbit anti-mouse Ig (Dakopatts, Copenhagen, Denmark) at 1/20 dilution. For direct immunofluorescence, spermatozoa were incubated with FITC-conjugated GB24 $\left(2.5 \mu \mathrm{g} / 100 \mu \mathrm{l} \mathrm{B} 2\right.$ medium), for $30 \mathrm{~min}$. Capacitation and the acrosome reaction were carried out at $37^{\circ} \mathrm{C}$ whereas the immunofluorescence tests were at room temperature. After final washes in PBS, samples were examined with a Zeiss Axiophot fluorescence microscope (sperm suspension and fixed slides of spermatozoa). Alternatively, sperm suspensions were analysed on a fluorescence-activated cell sorter (Odam, Brucker, ATC 3000, Wissembourg, France). Some samples of sperm suspensions were fixed with formaldehyde ( $1 \%$ in PBS) and stored at $4{ }^{\circ} \mathrm{C}$ to allow evaluation later.

Flow cytometric analysis of labelled spermatozoa was performed using samples containing $5 \times 10^{6}$ spermatozoa/ $\mathrm{ml}$ PBS. To distinguish spermatozoa from debris, a dot plot distribution of spermatozoa according to forward angle light scatter (correlating with cell size) and right angle light scatter (correlating with cell density) were used to determine a 'sperm window' as described by Haas \& Cunningham (1984). The fluorescence intensity of 10000 spermatozoa within the sperm window was computed by cytometry for each sample. A threshold was set so that the spermatozoa with a fluorescence intensity below this threshold were considered as negative and not represented.

Transmission electron microscopy. Electron microscopy was performed on 7 samples concurrently with immunofluorescence before and after induction of the acrosome reaction with A23187 (10 $\mu \mathrm{M}$ for $1 \mathrm{~h}$ ). Spermatozoa were fixed with $2.5 \%$ glutaraldehyde at $4^{\circ} \mathrm{C}$ for $1 \mathrm{~h}$, washed in $0.1 \mathrm{M}$-cacodylate buffer $\left(\mathrm{pH} \mathrm{7.4)}\right.$ ), post-fixed with $\mathrm{OsO}_{4}$, dehydrated in ethanol and embedded in Epon. Thin sections were post-stained with uranyl acetate and lead citrate (Nagae et al., 1986). Samples were examined with an Hitachi H600 electron microscope (75 kV).

Statistical analysis. The effects of A23187 in PBS and B2 medium as well as the effect of capacitation in B2 medium on the reactivity of GB24 on spermatozoa were compared using a variance analysis. A linear regression was computed from results obtained by flow cytometry and electron microscopy according to the least squares method.

\section{Results}

GB24 did not react with motile freshly ejaculated spermatozoa separated from seminal plasma (Fig. 1a,b). However, GB24 was positive on the acrosomal region of the head (Fig. 1c) of all spermatozoa after they were fixed onto slides and permeabilized with acetone. Almost $30 \%$ viable motile spermatozoa became strongly positive (Fig. 1d) after incubation for $6 \mathrm{~h}$ in B2 medium followed by chemical induction of the acrosome reaction by calcium ionophore A23187 (10 $\mu \mathrm{M}$ in B2 medium, 1/200 ethanol, 1 h). GB3 and IOT8, two control IgG1 monoclonal antibodies, did not react with spermatozoa under any condition. Spermatozoa from an infertile patient with more than 

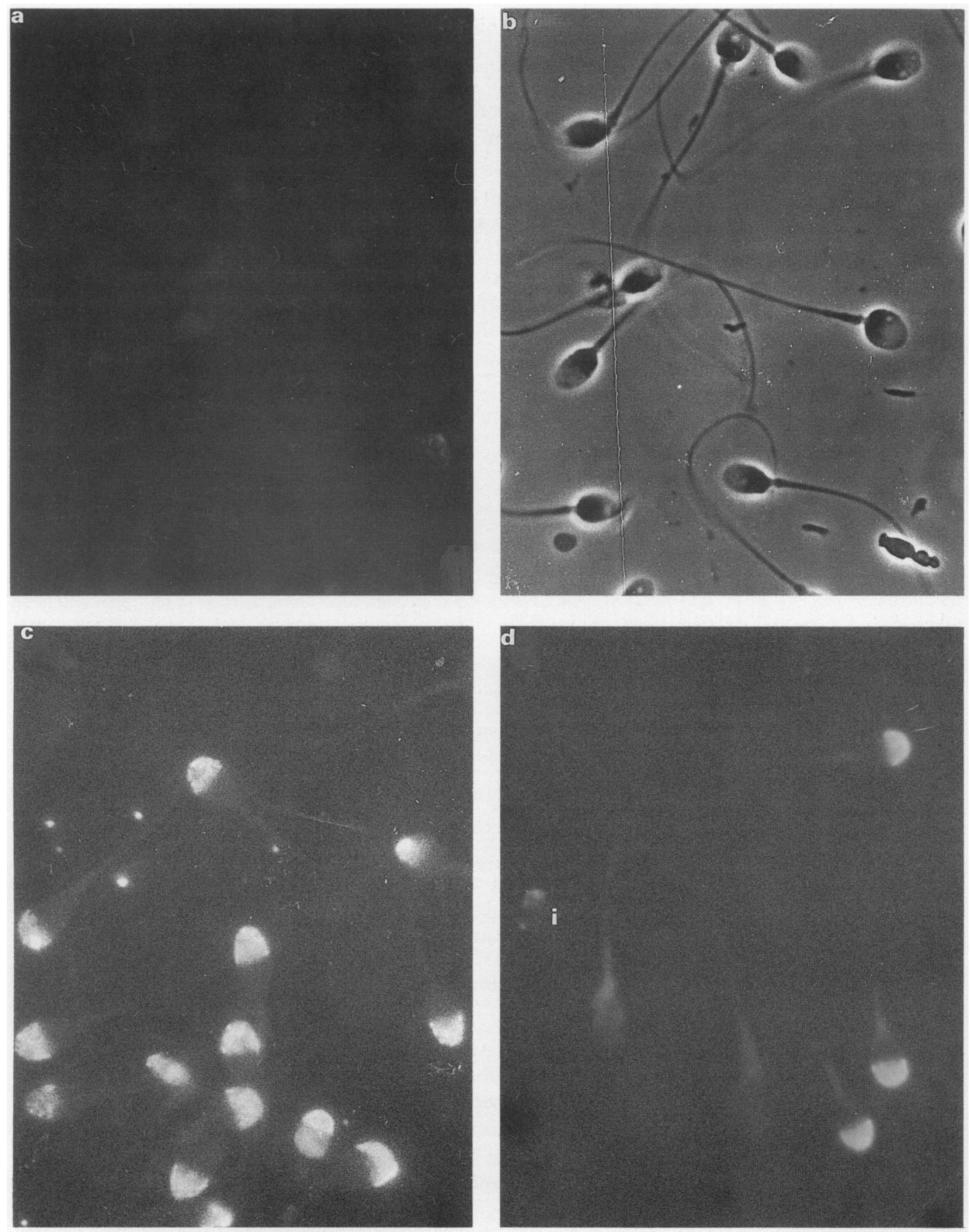

Fig. 1. Indirect immunofuoresence with monoclonal antibody GB24 on viable and fixed human spermatozoa. Most of the freshly ejaculated motile spermatozoa seen by phase-contrast microscopy (b) do not present any fluorescence (a). All spermatozoa, nevertheless, show an acrosomal fluorescence when fixed and permeabilized with acetone (c). Some spermatozoa also became positive after capacitation ( $6 \mathrm{~h}$ in $\mathrm{B} 2)$ following acrosome reaction induction $(10 \mu \mathrm{M}$ A23187. in B2. 1 h) (d). One acrosome was incompletely stained (i) and may correspond to an intermediate acrosome reaction stage. $\times 1000$. 
$90 \%$ microcephalic acrosomeless spermatozoa did not show any reactivity with GB24 even after permeabilization with acetone. A quantitative analysis of GB24 reactivity on spermatozoa was performed by flow cytometry at different stages of maturation. Uncapacitated spermatozoa from a donor showed 3\% positive for GB24 (Fig. 2a), and spermatozoa obtained after $6 \mathrm{~h}$ capacitation in B2 medium showed 5\% positive (Fig. 2b). A marked increase in the percentage of acrosomereacted spermatozoa (35\%) was observed (Fig. 2c) among capacitated spermatozoa incubated with A23187 $(10 \mu \mathrm{M}, 1 \mathrm{~h}$, ethanol 1/200). Ethanol alone (1/200 in B2 medium, $1 \mathrm{~h})$ did not modify the spontaneous percentage of reactivity obtained after $6 \mathrm{~h}$ capacitation (Fig. 2d). This effect of A23187 on GB24 reactivity was time-dependent (Fig. 3) and concentration-dependent (Fig. 4). The presence of calcium in the medium was necessary for the expression of antigen for GB24 on the surface of spermatozoa, because when A23187 was added in PBS instead of in B2 medium (Table 1) or in B2 medium with $2 \mathrm{mM}$-EGTA (data not shown) the reactivity of GB24 did not increase. Capacitation with B2 medium was also a prerequisite (Table 2).

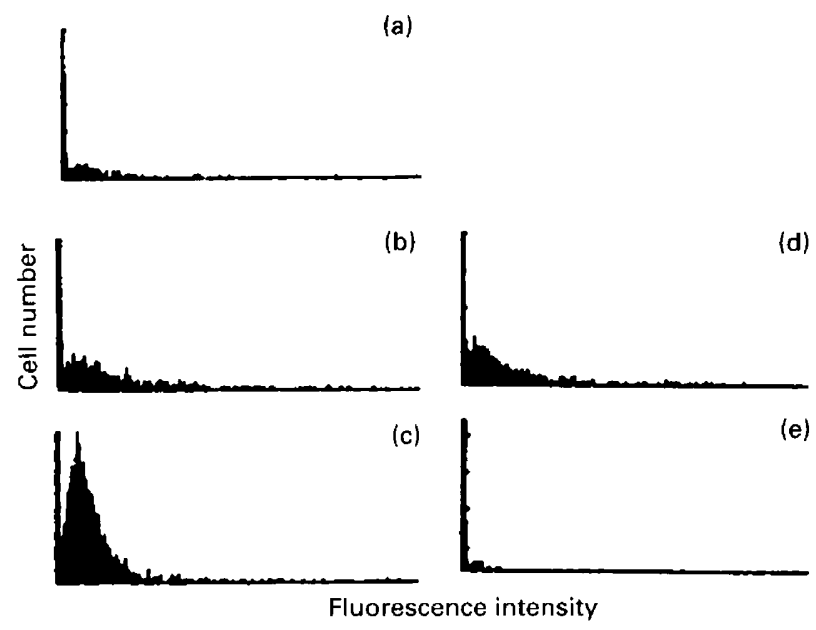

Fig. 2. Flow cytometric analysis of spermatozoa at different stages of maturation after direct immunofluorescence with GB24-FITC (see text). The percentage of stained cells was $3 \%$ for freshly ejaculated spermatozoa (a), 6\% for capacitated spermatozoa (6h in B2 medium) (b), $35 \%$ for A23187-treated ( $\mathrm{h}, 10 \mu \mathrm{M}$ in B2 medium) capacitated spermatozoa (c) and $1.5 \%$ for a control IgG1 monoclonal antibody, IOT8-FITC (e). Ethanol alone (1/200) in B2 medium (d) did not change the reactivity of capacitated spermatozoa.

These results suggested that the reactivity of GB24 on viable spermatozoa may be related to the acrosome reaction induced by A23187. We therefore carried out a direct comparison of the reactivity of GB24 to the acrosome reaction on spermatozoa evaluated by electron microscopy: 200 spermatozoa were counted in each pellet and classified as acrosome-reacted or not reacted. Only spermatozoa without any obvious morphological abnormalities were counted. Acrosome-reacted spermatozoa included total absence of plasma and outer acrosomal membranes on the anterior region of the sperm head with or without vesicles in the vicinity of the head. Spermatozoa with a degenerated acrosome were excluded as dead cells. The percentage of positive spermatozoa for GB24 measured by flow cytometry showed a direct correlation (Fig. 5) with the percentage of normal acrosome-reacted spermatozoa quantitated by electron microscopy after the same precapacitation in B2 medium with or without A23187 induction.

Indirect immunofluorescence, using GB24 and flow cytometry was then used as a standard test for evaluation of the A23187-induced acrosome reaction on motile capacitated spermatozoa of 9 proven fertile donors. All these spermatozoa presented normal characteristics and maintained a 


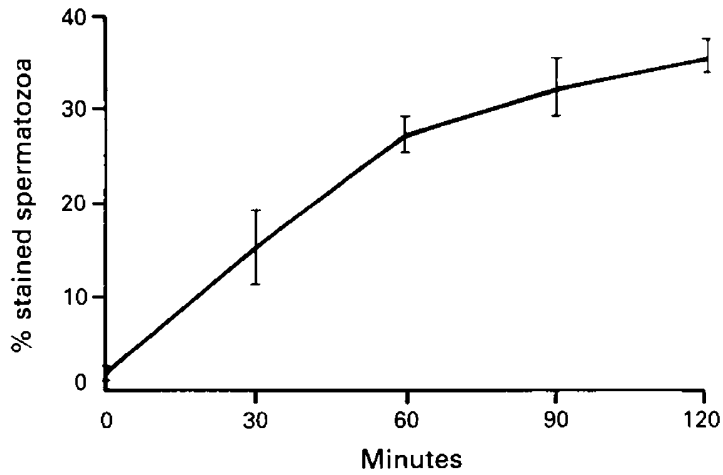

Fig. 3. Incubation of capacitated motile spermatozoa with $\mathrm{A} 23187(10 \mu \mathrm{M}$ in B2 medium, ethanol $1 / 200$ ) increased the reactivity to GB24 measured by flow cytometry after indirect immunofluorescence. A maximal response was obtained after $1 \mathrm{~h}$ of incubation. Ethanol alone $(1 / 200)$ in B2 medium did not modify the reactivity. GB3, an IgG1 monoclonal antibody also showed negative results. Values are the mean \pm s.d. of 3 different donors.

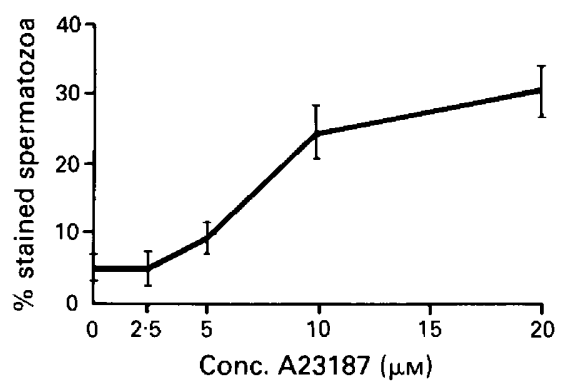

Fig. 4. Effect of A23187 concentration on the reactivity of GB24 against motile capacitated spermatozoa. Spermatozoa were incubated with A23187 in ethanol (1/200) in B2 medium for $1 \mathrm{~h}$. A maximal response was reached at a concentration of $10 \mu \mathrm{M}$. Incubations with ethanol alone (1/200 in B2 medium) or with an IgG1 monoclonal antibody GB3 showed negative results. Values are the mean \pm s.d. of 4 different donors.

Table 1. Role of calcium on the A23187-induced acrosome reaction of human spermatozoa as measured by indirect immunofluorescence (GB24-flow cytometry)

\begin{tabular}{lccc}
\hline Donors & $\begin{array}{c}\text { Fresh } \\
\text { ejaculated spermatozoa } \\
(\%)\end{array}$ & $\begin{array}{c}\text { A23187* } \\
\text { in PBS } \\
(\%)\end{array}$ & $\begin{array}{c}\text { A23187** } \\
\text { in B2 medium } \\
(\%)\end{array}$ \\
\hline 1 & $2 \cdot 5$ & 3 & 8 \\
2 & $3 \cdot 5$ & $5 \cdot 4$ & 15 \\
3 & 6 & 10 & 15 \\
4 & $2 \cdot 5$ & $3 \cdot 6$ & 15 \\
5 & $1 \cdot 3$ & 4 & 20 \\
Mean \pm s.d. & $3.4 \pm 1.9$ & $5 \cdot 5 \pm 2.7$ & $14.6 \pm 3.8^{* *}$ \\
\hline
\end{tabular}

Values are $\%$ of positive spermatozoa observed.

*A23187: $10 \mu \mathrm{M}, 1 \mathrm{~h}$.

$* * P<0.001$ (variance analysis). 
Table 2. Role of capacitation on the A23187induced acrosome reaction of human spermatozoa as measured by indirect immunofluorescence (GB24-flow cytometry)

\begin{tabular}{lcc}
\hline & $\begin{array}{c}\text { Without } \\
\text { capacitation } \\
(\%)\end{array}$ & $\begin{array}{c}\text { Capacitation in } \\
\text { B2 medium for 6h } \\
(\%)\end{array}$ \\
\hline Without A23187 & $4 \cdot 4 \pm 2 \cdot 0$ & $6 \cdot 8 \pm 2 \cdot 7$ \\
With A23187* & $7 \cdot 3 \pm 2 \cdot 2$ & $28 \cdot 2 \pm 4 \cdot 6^{* *}$ \\
\hline
\end{tabular}

Values are mean \pm s.d. of $\%$ positive spermatozoa observed from 4 donors.

*A23187: $10 \mu \mathrm{M}, 1 \mathrm{~h}$.

${ }^{* *} P<0.001$ (variance analysis).

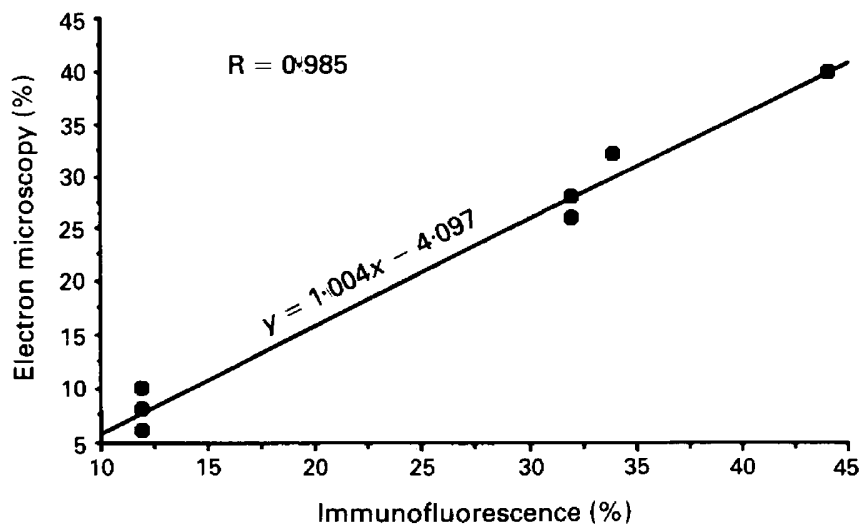

Fig. 5. Comparison of two methods of evaluation of the acrosome reaction. The percentage of labelled spermatozoa was determined by flow cytometry after indirect immunofluorescence with GB24. The same samples of capacitated and A23187-induced or not induced motile spermatozoa were prepared for electron microscopy. The percentages of acrosome-reacted spermatozoa were quantitated on 200 morphologically normal spermatozoa for each specimen. Spermatozoa with a complete absence of plasma and outer acrosomal membranes or the presence of vesicles of intermediate stage were counted as acrosome-reacted spermatozoa. Least squares method was used for statistical analysis.

good viability and motility during $6 \mathrm{~h}$ capacitation in B2 medium followed by $1 \mathrm{~h}$ incubation with $10 \mu \mathrm{M}-\mathrm{A} 23187$ (in B2 medium and ethanol, 1/200) (Table 3). When A23187 was added for the last hour of incubation, spermatozoa positive for GB24 increased from $\sim 5 \%$ to $32 \%$ with some between-donor variability (Table 3). The spermatozoa of one donor, who had an unexplained infertility, showed an absence of effect of A23187 (No. 10 in Table 3) although his spermatozoa maintained good motility during the test.

\section{Discussion}

The antigen recognized by the mouse monoclonal antibody GB24 on human trophoblast and lymphocytes (Hsi et al., 1988) is present on the anterior part of the human sperm head which corresponds to the acrosomal cap. This antigen cannot be detected by GB24 on freshly ejaculated 
Table 3. Reactivity to GB24 before and after A23187 induction of capacitated

( $6 \mathrm{~h}$ in $\mathrm{B} 2$ medium) viable spermatozoa

\begin{tabular}{|c|c|c|c|c|}
\hline \multirow[b]{2}{*}{ Patients } & \multicolumn{2}{|c|}{ Reactivity to GB24 (\%)† } & \multicolumn{2}{|c|}{ Motility $(\%) \ddagger$} \\
\hline & Before A23187 & After A23187* & Before A23187 & After A23187* \\
\hline 1 & $2 \cdot 6$ & 30 & 85 & 80 \\
\hline 2 & 7 & $30 \cdot 5$ & 75 & 70 \\
\hline 3 & 2 & 24 & 70 & 65 \\
\hline 4 & $1 \cdot 3$ & 29 & 80 & 80 \\
\hline 5 & 4 & 36 & 70 & 65 \\
\hline 6 & 6.5 & 35 & 80 & 75 \\
\hline 7 & $7 \cdot 5$ & 35 & 70 & 58 \\
\hline 8 & $3 \cdot 5$ & 22 & 75 & 70 \\
\hline 9 & 11 & 33 & 70 & 60 \\
\hline Mean \pm s.d. & $5 \cdot 2 \pm 2 \cdot 8$ & $32 \cdot 5 \pm 4 \cdot 6$ & 77 & 70 \\
\hline $10 \S$ & 7 & 8 & 85 & 80 \\
\hline
\end{tabular}

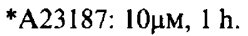

†Percentage of stained spermatozoa determined by flow cytometry.

$\ddagger$ Percentage of motile spermatozoa.

$\S U$ Unexplained infertility.

spermatozoa except after permeabilization by fixation with acetone. However, its reactivity appears on the surface of viable motile spermatozoa during maturation. The same conditions of dose-, time- and calcium-dependence needed for the ionophore A23187 to induce the acrosome reaction on viable spermatozoa (Jamil \& Whyte, 1981; Aitken et al., 1984; Byrd \& Wolf, 1986) were needed to increase GB24 reactivity. The evaluation of acrosome-reacted spermatozoa by electron microscopy correlated well with the reactivity of GB24 on spermatozoa. The acrosome reaction consists of an exocytotic loss of plasma membrane and outer acrosome membrane, as well as acrosomal matrix, and results in the externalization of the inner acrosomal membrane as shown with electron microscopy (Russell et al., 1979). Our results suggest that the antigen recognized by GB24 is present on the inner acrosome membrane and becomes accessible to the monoclonal antibody only after permeabilization of fixed spermatozoa or after the acrosome reaction of viable spermatozoa. Confirmation of this localization is now in progress in our laboratory using immuno-electron microscopy.

The antigen recognized by GB24 on human trophoblasts has been characterized (Hsi et al., 1988). It has been suggested that this antigen belongs to the polymorphic cross-reactive (TLX) antigens of trophoblast lymphocytes which may stimulate maternal responses to protect the allogeneically different fetus from immune assault by the maternal immune system. The antigens recognized by GB24 on spermatozoa and on trophoblast may be the same or share a common determinant. It is unlikely that this antigen on spermatozoa serves the same kind of protective function for spermatozoa, because the antigen is only expressed after the acrosome reaction. Therefore, during transportation of spermatozoa through the female genital tract, it is expected that this antigen is not expressed. Furthermore, the antigen on the spermatozoon does not show the same kind of polymorphism (data not shown) as that on the trophoblast (Hsi et al., 1988). McIntyre \& Faulk (1982) have found large amounts of TLX antigen in the seminal fluid and postulated that this antigen probably helps to pre-condition the uterus immunologically to accept the allogeneic embryo. Whether the expression of this antigen on spermatozoa after arrival in the vicinity of the oocyte does serve this function will need to be examined by further studies.

The use of A23187 was proposed as a valuable clinical test to evaluate the ability of motile spermatozoa to undergo a chemically induced acrosome reaction (Aitken et al., 1984). The antigen recognized by GB24 on the sperm head becomes accessible to the monoclonal antibody during and 
after an acrosome reaction induced by A23187. It thus represents a direct immunomarker of the acrosome reaction on viable spermatozoa. Such immunoassays have been already proposed by Byrd \& Wolf (1986) and Kallajoki et al. (1986). However, the monoclonal antibody used by these authors was raised against antigens of the matrix or the outer acrosomal membrane, which disappear after the acrosomal reaction, and so positive staining could only be obtained before the acrosome reaction on fixed spermatozoa with a permeabilized plasma membrane. In contrast, the reactivity of GB24 appears with viable spermatozoa during the acrosome reaction and, when used with flow cytometry, allows a direct quantitative evaluation of suspended cells. Although optimal conditions for a standard test have to be further defined, the use of GB24 as a direct, simple, clinical assay of the acrosome reaction on viable or fixed spermatozoa may contribute to the physiological and pathophysiological evaluation of sperm maturation.

We thank P. Chang from the Centre de Zoologie de Villefranche sur Mer for assistance with electron microscopy; Dr J.-Ph. Breittmayer for statistical analysis; Dr J. Sharps and Dr M. Fehlmann for helpful discussions; and C. Milesi-Fluet for secretarial assistance.

\section{References}

Aitken, R.J., Ross, A., Hargreave, T., Richardson, D. \& Best, F. (1984) Analysis of human sperm function following exposure to ionophore A23187. J. Androl. 5, 321-329.

Barros, C., Bedford, J.M., Franklin M.C. \& Austin, C.R. (1967) Membrane vesiculation as a feature of the mammalian acrosome reaction. $J$. Cell Biol..34, 1-5.

Bedford, J.M. (1983) Significance of the need for sperm capacitation before fertilization in eutherian mammals. Biol. Reprod. 28, 108-120.

Byrd, W. \& Wolf, D.P. (1986) Acrosomal status in fresh and capacitated human ejaculated sperm. Biol. Reprod. 34, 859-869.

Haas, G.G. \& Cunningham, M.S. (1984) Identification of antibody-laden sperm by cytofluorometry. Fert. Steril. 42, 606613.

Hsi, B.-L. \& Yeh, C.-J.G. (1986) Human villous trophoblasts studied by monoclonal antibodies GB25 and GB36. Placenta 5, 465-472.

Hsi, B.-L., Yeh, C.-J.G., Johnson, P.M., Bereford, N. \& Stern, P.L. (1987) Monoclonal antibody GBI7 recognizes human syncytiotrophoblast. $J$. Reprod. Immunol. 12, 235-244.

Hsi, B.-L., Yeh, C.-J.G., Fenichel, P., Samson, M. \& Grivaux, C. (1988) Monoclonal antibody GB24 recognizes a trophoblast-lymphocyte cross-reactive antigen. Am. J. Reprod. Immunol. Microbiol. 18, 21-27.

Jamil, K. \& White, I.G. (1981) Induction of acrosomal reaction in sperm with ionophore A23187 and calcium. Archs Androl. 7, 283-292.

Johnson, G.D. \& Holborow, E.J. (1986) Preparation and use of fluorochrome conjugates. In Handbook of Experimental Immunology, 4th edn, pp. 28.4-28.5, Ed. D. M. Weir. Blackwell Scientific Publications, Oxford.

Kallajoki, M., Virtanen, I. \& Suominen, J. (1986) The fate of acrosomal staining during the acrosome reaction of human spermatozoa as revealed by a monoclonal antibody and PNA-lectin. Int. J. Androl. 9, 181-194.

McIntyre, J.A. \& Faulk, W.P. (1982) Allotypic trophoblast-lymphocyte cross-reactive (TLX) cell surface antigens. Hum. Immunol. 4, 27-36.

Ménézo, Y. (1976) Milieu synthétique pour la survie et la maturation des gamètes et pour la culture de l'oeuf fécondé. C. r. hebd. Séanc. Acad. Sci. Paris, D 282, 1967-1970.

Nagae, T., Yanagimachi, R., Srivastava, N.P. \& Yanagimachi, H. (1986) Acrosome reaction in human spermatozoa. Fert. Steril. 45, 701-707.

Russell, L., Peterson, R.N. \& Freund, M. (1979) Morphologic characteristics of the chemically induced acrosome reaction in human spermatozoa. Fert. Steril. 32, 87-92.

Russo, C., Callegaro, L., Lanza, E. \& Ferrone, S. (1983) Purification of IgG monoclonal antibody by caprilic acid precipitation. J. Immunol. Methods 65, 269-271.

Talbot, P. \& Chacon, R.S. (1981) A triple-stain technique for evaluating normal acrosome reactions of human sperm. J. exp. Zool. 215, 201-208.

Verrando, P., Hsi, B.-L., Yeh, C.-J.G., Pisani, A., Seriyes, N. \& Ortonne, J.P. (1987) Monoclonal antibody GB3, a new probe for the study of human basement membranes and hemidesmosomes. Expl Cell Res. 170, 116-128.

Wolf, D.P., Boldt, J., Byrd, W. \& Bechtol, K.B. (1985) Acrosomal status evaluation in human ejaculated sperm with monoclonal antibodies. Biol. Reprod. 32, $1157-1162$.

Yanagimachi, R., Yanagimachi, H. \& Rogers, B.J. (1976) The use of zona-free animal ova as a test-system for the assessment of the fertilizing capacity of human spermatozoa. Biol. Reprod. 15, 471-476.

Received 9 March 1989 